\title{
Estudio de validación del Cuestionario de Afrontamiento del Estrés en personas trans de México
}

\author{
Lucía Quezada-Berumen, José Moral de la Rubia, Loren D. Ibarra-González y \\ Mónica T. González-Ramírez
}

Universidad Autónoma de Nuevo León, Nuevo León, México

Validation study of the Stress Coping Questionnaire with trans people from Mexico

\begin{abstract}
The Coping Strategies Questionnaire (CAE) is one of the most used instruments to evaluate coping strategies and styles in diverse populations; however, there is no evidence of its application in trans people, although this population is often discriminated, stigmatized, and presents social, occupational and health vulnerabilities. Therefor, the present study aimed to test factor structure of the CAE, prove internal consistency and convergent and discriminant validity of its factors, and describe the average profile of coping among trans people from Mexico. The sample consisted of 100 trans men and trans women, with ages ranging from 16 to 52 years, from different states of Mexico. The results showed that the CAE presents a factor structure different from that reported in other populations, with six first-order factors that show internal consistency and convergent and discriminant validity. The positive reappraisal factor was not defined, but was partially associated with the negative self-focusing factor. It is concluded that the CAE among trans people presents six first-order factors, with three higher-order underlying factors: negative emotional coping, social support and avoidant coping/religiosity.
\end{abstract}

Keywords: Coping; stress; factor structure; transsexual.

Resumen. El Cuestionario de Afrontamiento del Estrés (CAE) es uno de los instrumentos más usados para evaluar estrategias y estilos de afrontamiento; sin embargo, no se cuenta con evidencia sobre su aplicación en población trans, cuando ésta es objeto frecuente de discriminación, estigmatización y presenta diversas vulnerabilidades sociales, laborales y sanitarias. Así, este estudio tuvo como objetivos contrastar la estructura factorial del CAE, probar la consistencia interna y validez convergente y discriminante de sus factores, y describir el perfil promedio del afrontamiento en personas trans de México. La muestra se conformó por 100 hombres trans y mujeres trans con un rango de edad de 16 a 52 años de distintos estados de México. Los resultados mostraron que el CAE presenta una estructura factorial distinta a la reportada en otras poblaciones, con seis factores de primer orden que muestran consistencia interna y validez convergente y discriminante. El factor de reevaluación positiva no se definió, sino que parcialmente se asoció al de autofocalización negativa. Se concluye que el CAE en las personas trans presenta seis factores de primer orden a los que subyacen tres factores de orden superior: afrontamiento emocional negativo, apoyo social y afrontamiento evitativo/religiosidad.

Palabras clave: Afrontamiento; estrés; estructura factorial; transexual.

Recibido: 02 de agosto de 2017; aceptado: 22 de mayo de 2018 Correspondecia: Lucía Quezada-Berumen, Facultad de Psicología, Universidad Autónoma de Nuevo León, Nuevo León, México. Correo-e: luciaqb86@msn.com

Agradecimientos: A las 100 personas con identidad de género trans que participaron en el proceso de muestreo.

El estudio fue financiado por los autores quienes estaban adscritos a la Facultad de Psicología de la Universidad Autónoma de Nuevo León.

\section{Introducción}

Una persona trans es aquella que no se identifica con su sexo biológico o género asignado al nacer. Las personas trans pueden tener una expresión de género diferente a su sexo biológico asignado, así como también iniciar un tratamiento de reasignación para modificar de manera permanente su cuerpo (Barrios, 2014; Stryker, 2015). 
Al tener una identidad de género distinta a la asignada al nacer, las personas trans afrontan una serie de retos y condiciones adversas entre las que se encuentran: discriminación, exclusión social, educativa y laboral, disminución de sus derechos ciudadanos, falta de acceso y discriminación en el ámbito sanitario, así como alto grado de exposición a violencia transfóbica y crímenes de odio (Agius y Tobler, 2012; Peña, 2014).

Dichas condiciones pueden resultar en altos niveles de estrés psicológico para esta población, lo que repercute de manera significativa en su bienestar y calidad de vida. En este sentido, el estrés psicológico se define como la relación entre el individuo y su entorno, el cual es percibido por la propia persona como amenazante o desbordante de sus recursos (agobio, sobrecarga, irritabilidad) ante las dificultades presentes y que ponen en riesgo su bienestar (Cohen, Kamarck, y Mermelstein 1983). Cuando una situación se percibe como amenazante, de daño o pérdida, las personas responden de distintas maneras con el objetivo de reducir el malestar. No obstante, la reacción al estrés no está relacionada con la naturaleza objetiva del estímulo estresante, sino con la interpretación o significado psicológico que la persona asigna a las distintas situaciones, con su capacidad para controlar la situación estresante, y con la influencia de los patrones de conducta aprobados por la sociedad (Sandín 2003).

En este sentido, el afrontamiento se define como los esfuerzos cognitivos y conductuales que lleva a cabo el individuo para manejar las situaciones generadoras de estrés y adaptarse a ellas (Lazarus y Folkman, 1991). La persona no sólo evalúa lo que es estresante, sino que, una vez reconocida la fuente de estrés, la persona utiliza diversas formas para lidiar con éste (Peñacoba y Moreno, 1999); algunas pueden ser adaptativas y otras no (Sandín, 2003).

Lazarus y Folkman (1991) definen dos estilos de afrontamiento: 1) el enfocado al problema - estilo adaptativo-, el cual se utiliza con mayor frecuencia cuando la situación ha sido evaluada como susceptible de cambio, por lo que los individuos dirigen sus esfuerzos a la fuente de estrés para modificarla o eliminarla y así disminuir el malestar; 2) el enfocado a la emoción - estilo desadaptativo - , que tiene mayor probabilidad de utilizarse cuando se ha realizado una evaluación de que no puede hacerse nada para modificar la situación estresante del entorno y la persona únicamente encamina sus esfuerzos a regular los estados emocionales relacionados o producto de la situación estresante.

Existe un repertorio de instrumentos para evaluar el afrontamiento, entre los cuales se pueden mencionar la Escala de Modos de Afrontamiento de
Lazarus y Folkman (1991), el Inventario de Respuestas de Afrontamiento de Moos (1993), el COPE de Carver, Scheier y Weintraub (1989), el Indicador de Estrategia de Afrontamiento de Amirkhan (1990), el Inventario de Afrontamiento de Situaciones Estresantes de Endler y Parker (1990), y el Cuestionario de Afrontamiento del Estrés (CAE) de Sandín y Chorot (2003).

Entre las ventajas para considerar el CAE se encuentran que es un instrumento elaborado originalmente en español y ha evidenciado adecuadas propiedades psicométricas en población española (Sandín y Chorot, 2003), su número de ítems es accesible, y sus propiedades psicométricas han resultado ser adecuadas en estudios con muestras mexicanas (González y Landero 2007; Moral y Martínez, 2009; Moral y Miaja, 2012).

El CAE evalúa siete formas de afrontamiento establecidas a través de un análisis factorial con 592 participantes (Sandín y Chorot, 2003). Los factores de afrontamiento propuestos por el cuestionario son: Focalización en la Solución de Problemas (FSP), Autofocalización Negativa (AFN), Reevaluación Positiva (REP), Expresión Emocional Abierta (EEA), Evitación (EVT), Búsqueda de Apoyo Social (BAS) y Religión (RLG). Estas siete estrategias de afrontamiento se agrupan en dos factores de segundo orden superior, que corresponden a los estilos de afrontamiento planteados por Lazarus y Folkman (1991). Así, en el estilo de afrontamiento adaptativo se incluyen las estrategias de FSP, REP y BAS, mientras que en el estilo de afrontamiento desadaptativo, EEA, RLG, AFN y EVT (Sandín y Chorot, 2003). En el estudio de validación para México en estudiantes universitarios, realizado por González y Landero (2007), se encontró la misma estructura, exceptuando al factor RLG, el cual se incluyó dentro del estilo de afrontamiento adaptativo (enfocado al problema) en lugar del estilo desadaptativo (enfocado a la emoción). Con esta nueva configuración, los dos factores de segundo orden presentaron correlación negativa con una fuerza de asociación moderada en estudiantes universitarios (González y Landero, 2007), aunque resultaron independientes en padres de hijos con cáncer (Moral y Martínez, 2009) y en una muestra combinada de personas con VIH/SIDA y de población general (Moral y Miaja, 2012).

En México, Moral y Miaja (2012) estudiaron la consistencia interna y estructura factorial del CAE con una muestra combinada de 100 mujeres y 200 hombres seropositivos a VIH y 100 mujeres y 200 hombres de población general. Una vez reducido el cuestionario a 33 ítems, la estructura de siete factores de primer orden se reprodujo, así como los dos factores de segundo orden, 
salvo que RLG saturó en el factor de afrontamiento adaptativo, como en el estudio de González y Landero (2007). En esta muestra combinada, con estos 33 ítems retenidos la consistencia interna osciló de aceptable a buena en BAS, EEA, RLG y FSP, cuestionable en EVT y AFN, pero inaceptable en REP. Moral y Martínez (2009), en 15 parejas de padres y madres con hijos con diagnóstico de cáncer pediátrico, obtuvieron valores de consistencia interna de aceptables a buenos en BAS, FSP, RLG y REP, cuestionable en EVT y EEA, pero inaceptable en AFN. A su vez, González y Landero (2007) reportaron una consistencia interna de excelente a aceptable en BAS, EEA, RLG y FSP y cuestionable en EVT, AFN y REP.

Las personas trans se encuentran en una condición de mayor vulnerabilidad y adversidad a diferencia de la gran mayoría de las personas. Es importante mencionar que hay un limitado número de estudios en esta población en México (p.ej. Carrillo, 2008; Molina, Guzmán, y Martínez, 2015), siendo la mayoría estudios cualitativos. No se han detectado estudios en México de corte cuantitativo, ni realizados con muestras mayores a 50 participantes, que proporcionen información sobre alguna variable psicosocial o de salud mental en esta población. Lo anterior representa una importante área de oportunidad, así como una necesidad.

Debido a la importancia del concepto de afrontamiento en psicología de la salud y su aplicación a esta población vulnerable (Budge et al., 2013), contar con un instrumento que evalúe este concepto y que sea validado en población trans constituye un avance importante dentro de los estudios de género. Así, explorar las propiedades psicométricas del CAE en población trans proporcionará información relevante sobre la manera en que este grupo de personas hacen frente a la adversidad, lo que acrecienta el panorama de estudio dentro de los estudios de género y de la psicología. Se pretende que los resultados de este estudio sirvan como apoyo a futuras investigaciones, así como a los profesionales que trabajan en la mejora del bienestar y calidad de vida en esta población.

Por lo antes mencionado, el propósito de este estudio fue validar el CAE en personas trans de México para su aplicación en esta población, planteándose cuatro objetivos: 1) contrastar el modelo original de siete factores de primer orden jerarquizados a dos de orden superior correlacionados; 2) estimar la consistencia interna global y la consistencia interna de sus siete factores originales; 3 ) en caso de mal ajuste del modelo y problemas de consistencia interna en sus factores originales, explorar un nuevo modelo, estimando la consistencia interna y validez convergente y discriminante de sus factores; y 4) describir el perfil promedio de estrategias y estilos de afrontamiento en la muestra.

\section{Método}

\section{Participantes}

Se usó un muestreo no probabilístico, completándose una muestra intencional de 100 hombres trans y mujeres trans de distintos estados de la república mexicana. La muestra se obtuvo a través del procedimiento de bola de nieve. Los criterios de inclusión fueron: saber leer y escribir en español, poder acceder al cuestionario a través de internet, dar el consentimiento tras leer la información sobre el estudio para su inclusión en el mismo, informar de una identidad trans, e identificarse como mexicano. El incumplimiento de algún criterio de inclusión constituía criterio de exclusión. El único criterio de eliminación fue dejar alguna pregunta sin contestar.

Entre los 100 participantes del estudio, la media de edad fue de 25.86 años al momento de la evaluación ( $D T$ $=7.74$; rango $16-52$ ), y el tiempo promedio transcurrido desde que se reconoció por primera vez la identidad trans fue de 7.60 años $(D T=8.07$; rango $0-35)$.

El 21\% de la muestra informó su identidad de género como mujer trans $(n=21)$ con un promedio de edad de $30.33(D T=9.70$; rango $17-52)$ y un promedio de tiempo transcurrido desde que se reconoció por primera vez la identidad trans de 11.86 años ( $D T=10.92$; rango $1-35)$. Con respecto al tratamiento hormonal, $81 \%(\mathrm{n}=17)$ informó estar bajo tratamiento y $19 \%(n=4)$ reportó no haberlo iniciado.

El $79 \%$ de la muestra se identificó como hombre trans $(n=79)$, con un promedio de edad de $24.67(D T=$ 6.72; rango $16-50$ ), y un promedio de tiempo transcurrido desde que se reconoció por primera vez la identidad trans de 6.45 años $(D T=6.76$; rango $0-35)$. Con respecto al tratamiento hormonal, $55.4 \%(n=44)$ informó estar bajo tratamiento y $44.3 \%(n=35)$ reportó no haberlo iniciado.

\section{Instrumentos}

El Cuestionario de Afrontamiento al Estrés (CAE), de Sandín y Chorot (2003), está conformado por 42 afirmaciones en tiempo pasado, en las cuales la persona debe evaluar la frecuencia con la que utiliza cada una de ellas, marcando su respuesta en formato Likert $(0=$ nunca, $1=$ muy pocas veces, $2=$ a veces, $3=$ frecuentemente y $4=$ casi siempre). El cuestionario consta de siete escalas con una consistencia interna en general entre 
aceptable y excelente. Los coeficientes $\alpha$ de Cronbach para las siete escalas fueron: .64 para AFN, .71 para REP, .74 para EEA, .76 para EVT, .85 para FSP, .86 para RLG y .92 para BAS (Sandín y Chorot, 2003).

Se incluyeron preguntas sobre la identidad de género de los participantes (¿qué sexo se te asignó en tu acta de nacimiento original?, y ¿cuál es tu identidad de género hoy en día?), el tiempo transcurrido desde que se identificaron por primera vez como personas trans (¿edad en que reconociste tu identidad trans?, y ¿cuál es tu edad actual?) y si se encontraban o no en tratamiento hormonal (¿ya iniciaste tratamiento hormonal?).

\section{Procedimiento}

La encuesta se respondió en español a través de SurveyMonkey.com, difundiendo el enlace a través de redes sociales, siguiéndose un método de muestreo guiado por el participante o bola de nieve, donde a cada persona se le pedía invitar a otra a responder. La semilla inicial fueron dos hombres trans a quienes conocían directamente los investigadores. Todos los datos se trataron siguiendo los principios éticos de la American Psychological Association (2017).

\section{Análisis de datos}

En los análisis factoriales, a pesar de la escala de medida ordinal de los ítems, se usó la matriz de correlaciones de Pearson, en lugar de la matriz de correlaciones policóricas, por el tamaño reducido de la muestra, ya que la estimación de la correlación policórica requiere un tamaño de muestra mucho mayor (Lloret, Ferreres, Hernández, y Tomás, 2014).

Consecuente con la elección de matriz de correlaciones de Pearson, la consistencia interna global y de los factores se calculó con el coeficiente alfa de Cronbach $(\alpha)$. Aunque el coeficiente $\alpha$ varía en función del número de ítems de la escala (el valor de $\alpha$ es mayor cuando el número de ítems es elevado), a título orientativo puede interpretarase que valores de $\alpha<.50$ evidencian una consistencia interna inaceptable, de .50 a .59 pobre, de .60 a .69 cuestionable, de .70 a .79 aceptable, de .80 a .89 buena $\mathrm{y} \geq .90$ excelente (Cronbach y Shavelson, 2004).

Para comprobar la estructura del CAE se llevó a cabo el análisis factorial confirmatorio (AFC) y modelamiento de ecuaciones estructurales (MEE). La función de discrepancia se optimizó por Máxima Verosimilitud. Aunque este método requiere normalidad multivariada, proporciona estimaciones válidas con ítems tipo Likert si la curtosis multivariada estandarizada de Mardia (MSMK) es menor que 10 (Byrne, 2016), como fue el caso en la mayoría de los modelos (Tabla 1). Para compensar el hecho de no cumplirse de forma estricta con el supuesto de normalidad multivariada, que afecta esencialmente a la estimación de los errores típicos, éstos se calcularon por el método no paramétrico de percentiles corregidos de sesgo con la extracción de 2,000 muestras aleatorias (Byrne, 2016). Se consideraron ocho índices de ajuste. Siguiendo a Hooper, Coughlan y Mullen (2008) se interpretó como buen ajuste: $p$ para chi-cuadrado $\left(\chi^{2}\right)>.05$; chi-cuadrada relativa $\left(\chi^{2} /\right.$ $g l)<3$; índice de bondad de ajuste (GFI), índice de bondad de ajuste corregido (AGFI), índice de ajuste no normado (NNFI) e índice de ajuste comparativo (CFI) $\geq$ .95; y error cuadrático medio de aproximación (RMSEA) y residuo estandarizado cuadrático medio $($ SRMR $)<.07$. Además, se calculó la probabilidad por muestreo repetitivo de Bollen-Stine, la cual refleja bondad de ajuste con un valor mayor que .05 (Byrne, 2016). En un modelo, se rebasó por poco el valor de 10 en MSMK, pero en otro modelo la MSMK fue de 35.04, por lo que este modelo también se contrastó por mínimos cuadrados no ponderados.

Aparte de la significación de las estimaciones y bondad de ajuste, se evaluó la validez convergente (proporción de varianza de los indicadores explicada por su factor mayor que la atribuible a otros factores, entre ellos el azar). Esta se estableció por una varianza media extraída $(\mathrm{AVE})>.50$ y una fiabilidad compuesta o coeficiente omega de McDonald $(\omega)>.70$ (Malhotra y Dash, 2011). También, se valoró la validez discriminante de los factores, entendida como la proporción de varianza compartida entre dos factores menor que la proporción de varianza de los indicadores explicada por su factor y menor que dos tercios (Malhotra y Dash, 2011).

Para explorar un nuevo modelo factorial para el CAE, por problemas con el modelo original en la presente muestra, se usó el análisis factorial exploratorio (AFE), extrayéndose los factores por el método de Factorización de Ejes Principales, como sugieren Lloret et al. (2014). Se utilizó el método de rotación Promax con el fin de obtener una configuración más clara de los factores. Los factores se definieron desde la matriz de configuraciones con los ítems con las cargas más altas y de preferencia $>$ .40 o al menos > .30 (Lloret et al., 2014). El número de factores se determinó desde la matriz de correlaciones (producto-momento de Pearson) por el criterio de Velicer (media de la correlaciones parciales al cuadrado), el criterio de Velicer revisado (a la cuarta potencia), el análisis paralelo de Horn y coordenadas óptimas (percentil 95 en ambos análisis con 500 muestras generadas por permutación de datos brutos) y el criterio de Kaiser (autovalores mayores que uno), buscando la convergencia 
de criterios (Courtney, 2013). En el AFE de segundo orden se mantuvieron los mismos métodos.

Se compararon las medias usando el análisis de varianza de medidas repetidas y la prueba t de Student para dos muestras emparejadas. Se contrastó la normalidad distribucional por la prueba de D'Agostino-Pearson y la hipótesis de homocedasticidad e independencia de residuos por la prueba de Mauchly. Los cálculos estadísticos se realizaron con SPSS 24, módulo R 4.2 para SPSS24, AMOS 16 y Excel 2013. Se usó un nivel de significación o probabilidad exacta en los contrastes de .05 e intervalos de confianza para las estimaciones del $95 \%$.

\section{Resultados}

Contraste del modelo original de siete factores jerarquizados a dos de orden superior

Para estimar el modelo original (MO) se especificaron los dos factores de orden superior correlacionados; sin embargo, esta especificación generaba un problema de matriz definida negativa. Al eliminarse la correlación entre los dos factores, la solución convergió en 37 iteraciones, pero no fue admisible, al presentar dos varianzas de residuos estructurales negativas (en FSP y AFN). Cinco pesos estructurales y dos pesos de medida no fueron significativos, y los índices reflejaron mal ajuste, salvo la chi-cuadrada relativa (Tabla 1).

\section{Consistencia interna de los factores originales}

Al estimar la consistencia interna de los siete factores de primer orden esperados (cada uno de ellos definido por seis ítems), los valores del coeficiente alfa de Cronbach oscilaron de inaceptables a muy buenos. Los análisis indicaron que el ítem 22 de FSP, el ítem 16 de AFN, los ítems 17, 24, 31 y 38 de REP, el ítem 39 de EEA y el ítem 40 de EVT, afectaban el coeficiente $\alpha$ de Cronbach de sus respectivos factores y su eliminación mejoraba dicho indicador. Con una versión reducida de la escala conformada por 34 ítems, la consistencia interna de los factores osciló de cuestionable a excelente, y la global fue aceptable (Tabla 2).

Tabla 1. Índices de bondad de ajuste

\begin{tabular}{|c|c|c|c|c|c|c|c|c|c|c|c|}
\hline \multirow{2}{*}{$\begin{array}{l}\text { Índices } \\
\text { de ajuste }\end{array}$} & \multicolumn{2}{|c|}{ Interpretación } & \multicolumn{9}{|c|}{ Modelos } \\
\hline & Bueno & Malo & MO & $6 \mathrm{FJ}-3 \mathrm{FC}$ & $\mathrm{M} 2 \mathrm{O}$ & AFN & EEA & EVT & FSP & RLG & BAS \\
\hline$\chi^{2}$ & & & 1485.763 & 935.578 & 14.084 & 17.262 & 4.229 & 5.998 & 3.273 & 16.284 & 11.170 \\
\hline$g l$ & & & 812 & 519 & 9 & 12 & 4 & 4 & 3 & 6 & 8 \\
\hline$p$ & $>.05$ & $<.01$ & $<.001$ & $<.001$ & .119 & .140 & .376 & .199 & .351 & .012 & .192 \\
\hline$p$ de B-S & $>.05$ & $<.01$ & $<.001$ & $<.001$ & .159 & .258 & .550 & .287 & .576 & .382 & .487 \\
\hline$\chi^{2} / g l$ & $\leq 2$ & $>3$ & 1.83 & 1.803 & 1.565 & 1.439 & 1.057 & 1.499 & 1.091 & 2.714 & 1.396 \\
\hline GFI & $\leq .95$ & $\leq .85$ & .615 & .676 & .954 & .956 & .983 & .978 & .988 & .945 & .968 \\
\hline AGFI & $\leq .90$ & $\leq .80$ & .572 & .628 & .892 & .898 & .935 & .918 & .938 & .808 & .915 \\
\hline NNFI & $\leq .95$ & $\leq .85$ & .663 & .763 & .833 & .952 & .997 & .942 & .988 & .947 & .983 \\
\hline CFI & $\leq .95$ & $\leq .85$ & .682 & .774 & .900 & .973 & .999 & .977 & .999 & .979 & .991 \\
\hline RMSEA & $\leq .07$ & $\leq .10$ & .092 & .090 & .076 & .067 & .024 & .071 & .030 & .132 & .063 \\
\hline SRMR & $\leq .07$ & $\leq .10$ & .141 & .129 & .071 & .047 & .030 & .052 & .021 & .046 & .029 \\
\hline MMK & & & 116.066 & 125.540 & 6.009 & 7.133 & 9.507 & 1.189 & 6.988 & 68.670 & 19.600 \\
\hline MSMK & $<2$ & $<10$ & 9.546 & 12.334 & 3.067 & 3.177 & 5.681 & 0.710 & 4.176 & 35.043 & 10.002 \\
\hline
\end{tabular}

Notas. Índices de ajuste: $\chi^{2}=$ valor mínimo de la función de discrepancia o estadístico chi-cuadrado, $g l=\operatorname{grados}$ de libertad, $p=$ probabilidad o nivel de significación exacto en un contraste unilateral bajo una distribución chi-cuadrado, $p$ de $\mathrm{B}-\mathrm{S}=$ probabilidad por muestreo repetitivo de Bollen-Stine con la extracción de 2.000 muestras aleatorias, $\chi^{2} / \mathrm{gl}=$ chi-cuadrada relativa, GFI $=$ índice de bondad de ajuste, AGFI = índice de bondad de ajuste corregido, NNFI = índice de ajuste no normado, CFI = índice comparativo de ajuste, RMSEA = error de aproximación cuadrático medio, SRMR = residuo estandarizado cuadrático medio, MMK = curtosis multivariada de Mardia, MSMK = curtosis multivariada estandarizada de Mardia. Modelos: $\mathrm{MO}=$ modelo original contrastado con siete factores jerarquizados a dos factores independientes, $6 \mathrm{FJ}-3 \mathrm{FC}=$ Modelo de seis factores jerarquizados a tres factores correlacionados, $\mathrm{M} 2 \mathrm{O}=$ modelo con un factor de afrontamiento emocional con tres indicadores (FSP, AFN y EEA) y tres variables exógenas (EVT, RLG y BAS), estando correlacionadas EVT y RLG, y especificándose dos vías estructurales: de BAS hacia EEA y de EVT hacia FSP (Figura 1). 
Análisis factorial exploratorio de primer orden y consistencia interna y validez convergente $y$ discriminante de los factores

Debido al mal ajuste del modelo original se exploró uno nuevo. La matriz de correlaciones entre los 42 ítems se mostró apta para la extracción de factores. La medida de adecuación muestral de Kaiser, Meyer y Olkin fue mayor $.50(\mathrm{KMO}=.68)$ y se rechazó la hipótesis nula de la equivalencia de la matriz de correlaciones a una matriz identidad por la prueba de esfericidad de Bartlett $\left(\chi_{[861]}^{2}=2,532.79, p<.001\right)$. El análisis paralelo de Horn, coordenadas óptimas y la media mínima de las correlaciones parciales a la cuarta potencia convergieron en cinco factores. La media mínima de las correlaciones parciales al cuadrado indicó seis factores y el criterio de Kaiser 12. Al forzar la solución a siete factores, conforme con la expectativa, se explicó el $52.5 \%$ de la varianza total. Tras la rotación oblicua de la matriz factorial, los factores BAS (ítems 6, 13, 20, 27, 34 y 41), FSP (ítems 1, 8, 15, 22, 29 y 36), RLG (ítems 7, 14, 21, 28, 35 y 42), EEA (ítems 4, 11, 18, 25 y 32) y EVT (ítems 5, 12, 19, 26 y 33) se reprodujeron, pero no así los factores REP y AFN. El factor AFN se desdobló (ítems 2, 9 y 23 en el quinto factor e ítems 16,30 y 37 en el séptimo) y el factor REP se perdió. Los ítems 3 y 10 de REP saturaron en el factor de AFN (ítems 2, 9 y 23 de la escala orinal de AFN). Los ítems 17, 24, 31, 38, 39 y 40 no saturaron en los factores esperados. Los ítems 16 y 22, aunque cargaron en el factor esperado, tuvieron comunalidades en la extracción menores que $.25 \mathrm{y}$ cargas en las matrices de patrones y estructural menores que .40. Los ítems 22 y 33 saturaron en el factor esperado, tuvieron comunalidades en la extracción mayores que .25 y cargas mayores que .30 , pero menores que .40. Los restantes ítems saturaron en el factor esperado con cargas mayores que .40 y comunalidades mayores que .25 (Tabla 3). Cabe señalar que los ítems $16,17,22,24,31,38,39$ y 40 también presentaron problemas de configuración factorial y comunalidades menores que .25 en los modelos de seis (criterio de Velicer) y cinco factores (análisis paralelo, coordenadas óptimas y criterio de Velicer revisado).

A continuación se eliminaron los ocho ítems que generaban una disminución en la consistencia interna de su factor esperado (Tabla 2) y mostraron problemas en la matriz de configuraciones de siete (Tabla 3 ), seis y cinco factores (ítems 16, 17, 22, 24, 31, 38, 39 y 40). Con los 34 ítems restantes se procedió a realizar el AFE de primer orden. La medida de adecuación muestral de Kaiser, Meyer y Olkin $(\mathrm{KMO}=.73)$ y la prueba de esfericidad de Bartlett $\left(\chi_{[561]}^{2}=2094.37, p<.001\right)$, mostraron que la matriz de correlaciones era adecuada para extraer factores (Lloret et al., 2014).

Se obtuvieron ocho factores con autovalores $\geq 1$. La media mínima de las correlaciones parciales elevadas a la cuarta potencia (criterio revisado de Velicer) indicó siete factores, conforme a la expectativa. Por la media mínima de las correlaciones parciales elevadas al cuadrado (criterio de Velicer) fue seis, y por el análisis paralelo de Horn y coordenadas óptimas fue cinco. Ante esta discrepancia se optó por el criterio revisado de Velicer que coincide con la expectativa. Al extraer los siete factores, se explicó el $67.3 \%$ de la varianza total.

Tabla 2. Consistencia interna del cuestionario y sus escalas

\begin{tabular}{lccc}
\hline Escalas & $\alpha$ de Cronbach (42 ítems) & Ítems eliminados & $\alpha$ de Cronbach (34 ítems) \\
\hline Escala Total & .81 & $16,17,22,24,31,38,39$ y 40 & .79 \\
Focalización en la Solución de Problemas (FSP) & .84 & 22 & .88 \\
Autofocalización Negativa (AFN) & .74 & 16 & .76 \\
Reevaluación Positiva (REP) & .40 & $39,24,31,38$ & .70 \\
Expresión emocional abierta (EEA) & .79 & 40 & .83 \\
Evitación (EVT) & .66 & - & .68 \\
Búsqueda de Apoyo Social (BAS) & .90 & -91 & .90 \\
Religión (RLG) & .91 & \\
\hline
\end{tabular}

Notas. ítems eliminados 22. Hablé con las personas implicadas para encontrar una solución al problema; 16. Comprendí que yo fui el principal causante del problema; 17. Descubrí que en la vida hay cosas buenas y gente que se preocupa por los demás; 24 . Comprendí que otras cosas, diferentes del problema, eran para mí más importantes; 31 . Experimenté personalmente eso de que «no hay mal que por bien no venga»; 38. Comprobé que, después de todo, las cosas podían haber ocurrido peor; 39. Luché y me desahogué expresando mis sentimientos; 40. Intenté olvidarme de todo. 
Tras la rotación oblicua de la matriz factorial, se definió un primer factor conformado por los seis ítems de RLG (ítems 7, 14, 21, 28, 35 y 42); uno segundo por los seis ítems de BAS (ítems 6, 13, 20, 27, 34 y 41); uno tercero por los cinco ítems de AFN (ítems 2, 9, 23, 30 y 37 ) y dos de REP (ítems 3 y 10); uno cuarto por los cinco ítems de FSP (ítems 1, 8, 15, 29 y 36); el quinto por los cinco ítems de EEA (ítems 4, 11, 18, 25 y 32); el sexto por los ítems $5,12,19,26$ y 33 correspondientes a EVT, y finalmente el séptimo conformado sólo por el ítem 35 de RLG, siendo esta su segunda carga factorial más alta. La solución de siete factores se descartó, debido a la insuficiencia de indicadores para el último factor, y se forzó la solución a seis factores, conforme al criterio de Velicer.

Al extraer los seis factores se explicó el $63.8 \%$ de la varianza total. Tras la rotación, se encontró un primer factor conformado por los seis ítems de RLG, el segundo por los seis ítems de BAS, el tercero por los cinco ítems de FSP y el cuarto por los cinco ítems de AFN (en el que saturaron algunos items de reevaluación positiva). El quinto factor se constituyó por los cinco ítems de EEA y el sexto por los cinco ítems de EVT. Los factores RLG, BAS, FSP y EEA presentaron validez convergente (AVE $>.50$ y $\omega>.70)$, al igual que AFN (AVE > .40 y $\omega>.80$ ); no obstante, EVT no cumplió con los dos criterios de validez convergente (Tabla 4). La varianza máxima compartida entre los factores fue del $18.3 \%$, por lo que contaron con validez discriminante.

\section{Análisis factorial exploratorio de segundo orden}

Las puntuaciones en los factores se obtuvieron sumando los ítems, invirtiéndose las puntuaciones de los ítems 3 y 10 en AFN, y dividiendo por el número de ítems sumados, para obtener un rango continuo y homogéneo de 0 a 4 . El análisis paralelo de Horn, el de coordenadas óptimas, los criterios de Velicer y el criterio de Kaiser, convergieron en tres factores; por tanto, se extrajeron tres factores de segundo orden.

Al extraer tres factores se explicó el $70.2 \%$ de la varianza total. Tras la rotación, se configuró un primer factor con FSP, AFN y EEA, su consistencia interna fue buena ( $\alpha=.87$ entre los 17 ítems de las tres escalas), y se le denominó afrontamiento emocional negativo (AEN). El segundo factor quedó definido por BAS y EEA (su segunda carga más alta y mayor que .30 fue en este factor); su consistencia interna fue excelente ( $\alpha=.90$ entre los 11 ítems de las dos escalas), y se le denominó apoyo social (ASO). Finalmente, el tercer factor se compuso de EVT y RLG, su consistencia interna fue buena $(\alpha=.81$ entre los 11 ítems de las dos escalas), y se le denominó afrontamiento evitativo y religiosidad (AER). Sólo la correlación entre los factores de AEN y AER fue significativa $\left(r_{[100]}=-.25, p=.013\right)$. Así, la varianza máxima compartida fue $6.1 \%$, la cual fue menor que el AVE de cada uno de los tres factores y menor que $67 \%$, por lo que éstos contaron con validez discriminante. No obstante, ninguno de los tres factores satisfizo los dos criterios de validez convergente: $\mathrm{AVE}=.40 \mathrm{y} \omega=.66 \mathrm{en}$ $\mathrm{AEN}, \mathrm{AVE}=.40 \mathrm{y} \omega=.55$ en $\mathrm{ASO}, \mathrm{y} \mathrm{AVE}=.37 \mathrm{y} \omega=$ .54 en AER.

Al forzar la solución a dos factores, conforme a la expectativa, se explicó el $51 \%$ de la varianza total. El primer factor quedó definido por AFN y EEA, su consistencia interna fue buena $(\alpha=.83$ entre los 12 ítems de las dos escalas) y se le denominó afrontamiento emocional negativo (AEN). El segundo quedó configurado por FSP y BAS, su consistencia interna también fue buena $(\alpha=.85$ entre los 11 ítem de las dos escalas) y se le denominó afrontamiento activo (AA). Bajo esta estructura de segundo orden, EVT y RLG obtuvieron cargas configuracionales y estructurales bajas $(<.30)$. La varianza compartida entre los dos factores fue de $20.4 \%$, que es un valor menor al AVE de cada factor y menor que $67 \%$, por lo que ambos factores contaron con validez discriminante. No obstante, ninguno de los dos factores satisfizo los dos criterios de validez convergente: $\mathrm{AVE}=.38$ y $\omega=.54$ en $\mathrm{AEN}, \mathrm{y} \mathrm{AVE}=.48$ y $\omega=.59$ en $\mathrm{AA}$.

\section{Análisis factorial confirmatorio del modelo revisado y su reformulación}

Se buscó confirmar la estructura factorial encontrada en el AFE de segundo orden, con seis factores jerarquizados a tres factores de orden superior correlacionados (Modelo 6FJ-3FC). En este modelo, dos correlaciones entre los factores de segundo orden y dos pesos estructurales no resultaron significativos tanto por ML como por percentiles corregidos de sesgo (PCS), y los índices de bondad de ajuste fueron malos, salvo la chi-cuadrada relativa (Tabla 1).

Se optó por definir modelos unifactoriales para cada una de las seis escalas y un modelo de ecuaciones estructurales para la relación entre los factores. El modelo de un factor con siete indicadores (ítems 2, 3, 9, 10, 23, 30 y 37) para medir AFN tuvo ocho índices de ajuste buenos y uno aceptable una vez que se liberaron dos correlaciones (entre el residuo del ítem 30 y los residuos de los ítems 23 y 37 ), presentó todas sus estimaciones significativas por PCS y cumplió con el criterio de fiabilidad compuesta para validez convergente $(\omega=.81)$, pero no con el de la varianza media extraída $(\mathrm{AVE}=.39)$. El modelo de un factor con cinco indicadores (ítems 4,11 , 
Tabla 3. Comunalidades en la extracción y cargas en la matriz de configuraciones

\begin{tabular}{|c|c|c|c|c|c|c|c|c|c|}
\hline \multirow{2}{*}{ Ítems } & \multirow{2}{*}{ FE } & \multirow{2}{*}{ Com. } & \multicolumn{7}{|c|}{ Cargas en la matriz de configuraciones } \\
\hline & & & F1 & $\mathrm{F} 2$ & F3 & F4 & F5 & F6 & F7 \\
\hline 34 & BAS & .74 & .89 & .09 & -.21 & -.04 & .02 & .03 & -.12 \\
\hline 27 & BAS & .72 & .82 & -.10 & .03 & -.15 & .21 & -.07 & .10 \\
\hline 13 & BAS & .63 & .80 & .02 & -.01 & -.05 & -.12 & .20 & -.01 \\
\hline 41 & BAS & .69 & .76 & -.08 & .10 & .08 & .17 & -.06 & .03 \\
\hline 20 & BAS & .70 & .76 & .10 & .03 & .08 & .05 & -.04 & .14 \\
\hline 6 & BAS & .59 & .66 & .01 & .13 & .04 & -.21 & -.17 & .01 \\
\hline 39 & EEA & .35 & .47 & .02 & .14 & .09 & -.17 & -.12 & .14 \\
\hline 17 & REP & .36 & .46 & -.10 & .12 & .05 & -.25 & .05 & -.16 \\
\hline 31 & REP & .10 & .19 & .03 & -.11 & .11 & -.18 & .09 & .08 \\
\hline 21 & RLG & .84 & -.03 & .90 & .09 & .01 & .20 & -.01 & -.12 \\
\hline 42 & RLG & .73 & -.04 & .87 & -.02 & .06 & .03 & -.06 & -.07 \\
\hline 14 & RLG & .76 & .06 & .86 & .06 & -.02 & .16 & -.11 & -.05 \\
\hline 7 & RLG & .66 & .07 & .79 & -.01 & -.21 & -.01 & -.09 & .01 \\
\hline 35 & RLG & .60 & -.03 & .73 & -.09 & .04 & -.22 & -.04 & .18 \\
\hline 28 & RLG & .62 & .01 & .70 & -.11 & .06 & -.32 & .13 & .10 \\
\hline 36 & FSP & .63 & -.01 & -.01 & .87 & .04 & .08 & -.03 & .28 \\
\hline 15 & FSP & .74 & .11 & -.06 & .81 & -.01 & .10 & .01 & -.15 \\
\hline 29 & FSP & .74 & .09 & .08 & .76 & -.01 & -.01 & .05 & -.12 \\
\hline 8 & FSP & .62 & -.06 & .02 & .71 & -.02 & -.10 & -.02 & -.08 \\
\hline 1 & FSP & .58 & -.07 & -.07 & .64 & -.20 & -.27 & -.06 & .37 \\
\hline 22 & FSP & .22 & .25 & .02 & .36 & .06 & .08 & -.06 & .05 \\
\hline 11 & EEA & .72 & .13 & .02 & -.05 & .80 & .09 & .10 & -.13 \\
\hline 25 & EEA & .50 & -.05 & -.02 & .10 & .78 & .06 & .06 & -.21 \\
\hline 4 & EEA & .51 & -.08 & .02 & -.01 & .72 & .01 & -.09 & .02 \\
\hline 32 & EEA & .64 & .02 & -.14 & -.04 & .67 & .02 & -.15 & .18 \\
\hline 18 & EEA & .45 & .07 & .07 & -.11 & .57 & -.12 & -.13 & .12 \\
\hline 24 & REP & .19 & -.04 & -.14 & .31 & .32 & -.07 & .17 & .04 \\
\hline 9 & AFN & .59 & .14 & .04 & .02 & -.04 & .68 & .29 & .12 \\
\hline 3 & REP & .40 & .06 & .04 & -.07 & .01 & -.65 & .01 & -.03 \\
\hline 2 & AFN & .52 & -.09 & -.03 & .02 & .06 & .56 & -.19 & .26 \\
\hline 10 & REP & .61 & .15 & .04 & .23 & -.06 & -.53 & .11 & -.16 \\
\hline 23 & AFN & .53 & .11 & .03 & -.15 & .07 & .48 & .01 & .28 \\
\hline 5 & EVT & .50 & .07 & -.19 & -.10 & -.12 & -.01 & .72 & .04 \\
\hline 26 & EVT & .54 & -.02 & -.06 & -.14 & -.07 & -.03 & .71 & .16 \\
\hline 12 & EVT & .47 & -.15 & .05 & .19 & .06 & .13 & .61 & -.06 \\
\hline 19 & EVT & .34 & -.10 & .24 & .19 & .17 & .02 & .32 & .20 \\
\hline 33 & EVT & .27 & -.03 & .25 & .22 & .06 & -.04 & .31 & .06 \\
\hline 37 & AFN & .43 & -.05 & .03 & .10 & -.05 & .17 & .04 & .63 \\
\hline 38 & REP & .33 & .12 & -.02 & -.08 & -.03 & .13 & .01 & .49 \\
\hline 30 & AFN & .39 & .03 & .08 & .02 & -.07 & .32 & .12 & .47 \\
\hline 16 & AFN & .19 & .06 & -.05 & .22 & -.02 & .05 & .04 & .34 \\
\hline 40 & EVT & .32 & .04 & -.02 & -.22 & .13 & -.04 & .25 & .33 \\
\hline
\end{tabular}

Notas. Método de extracción: factorización de eje principal. Método de rotación: Promax con normalización Kaiser.

La rotación convergió en 7 iteraciones. $\mathrm{FE}=$ Factor esperado: $\mathrm{AFN}=$ autofocalización negativa, BAS = búsqueda de apoyo social, EEA = expresión emocional abierta, EVT = evitación, FSP = focalización en la solución de problemas, REP = reevaluación positiva y RLG $=$ religión. Com. $=$ comunalidad en la extracción. Se destacan las cargas que definen el factor esperado: en negrilla las que son $>.40$ y en cursiva las que son $>.30$ 
Tabla 4. Cargas configuracionales y estructurales de los ítems y consistencia interna y validez convergente de los factores

\begin{tabular}{|c|c|c|}
\hline Ítems* & $c c$ & ce \\
\hline \multicolumn{3}{|l|}{ Religión $(\alpha=.91 ; \mathrm{AVE}=.64$ y $\omega=.95)$} \\
\hline 21. Acudí a la Iglesia para rogar que se solucionase el problema & .87 & .88 \\
\hline 42. Acudí a la Iglesia para poner velas o rezar & .87 & .85 \\
\hline 14. Pedí ayuda espiritual a algún religioso (sacerdote, etc.) & .86 & .84 \\
\hline 7. Asistí a la Iglesia & .80 & .78 \\
\hline 35. Recé & .74 & .73 \\
\hline 28. Tuve fe en que Dios remediaría la situación & .71 & .72 \\
\hline \multicolumn{3}{|l|}{ Búsqueda de Apoyo Social $(\alpha=.90 ;$ AVE $=.63$ y $\omega=.95)$} \\
\hline 34. Pedí a algún amigo o familiar que me indicara cuál sería el mejor camino a seguir & .92 & .87 \\
\hline 27. Hablé con amigos o familiares para que me tranquilizaran cuando me encontraba mal & .82 & .81 \\
\hline 41. Procuré que algún familiar o amigo me escuchase cuando necesité manifestar mis sentimientos & .81 & .79 \\
\hline 13. Pedí consejo a algún pariente o amigo para afrontar mejor el problema & .77 & .77 \\
\hline 20. Pedí a parientes o amigos que me ayudaran a pensar acerca del problema & .72 & .83 \\
\hline 6. Le conté a familiares o amigos cómo me sentía & .61 & .66 \\
\hline \multicolumn{3}{|l|}{ Focalización en la Solución de Problemas $(\alpha=.88 ; \mathrm{AVE}=.60$ y $\omega=.93)$} \\
\hline 36. Pensé detenidamente los pasos a seguir para enfrentarme al problema & .88 & .76 \\
\hline 15. Establecí un plan de actuación y procuré llevarlo a cabo & .85 & .85 \\
\hline 29. Hice frente al problema poniendo en marcha varias soluciones concretas & .76 & .84 \\
\hline 8. Traté de solucionar el problema siguiendo unos pasos bien pensados & .74 & .79 \\
\hline 1. Traté de analizar las causas del problema para poder hacerle frente & .57 & .62 \\
\hline \multicolumn{3}{|l|}{ Autofocalización negativa $(\alpha=.82 ;$ AVE $=.40$ y $\omega=.88)$} \\
\hline 9. No hice nada concreto puesto que las cosas suelen ser malas & .73 & .69 \\
\hline 23. Me sentí indefenso/a e incapaz de hacer algo positivo para cambiar la situación & .66 & .74 \\
\hline 3. Intenté centrarme en los aspectos positivos del problema & -.66 & .60 \\
\hline 2. Me convencí de que hiciese lo que hiciese las cosas siempre me saldrían mal & .64 & .72 \\
\hline 10. Intenté sacar algo positivo del problema & -.63 & .65 \\
\hline 30. Me di cuenta de que por mí mismo no podía hacer nada para resolver el problema & .55 & .54 \\
\hline 37. Me resigné a aceptar las cosas como eran & .43 & .69 \\
\hline \multicolumn{3}{|l|}{ Expresión Emocional Abierta $(\alpha=.83 ; \mathrm{AVE}=.51$ y $\omega=.90)$} \\
\hline 4. Descargué mi mal humor con los demás & .75 & .72 \\
\hline 11. Insulté a ciertas personas & .73 & .80 \\
\hline 32. Me irrité con alguna gente & .69 & .74 \\
\hline 25. Agredí a algunas personas & .68 & .63 \\
\hline 18. Me comporté de forma hostil con los demás & .65 & .66 \\
\hline \multicolumn{3}{|l|}{ Evitación $(\alpha=.68 ;$ AVE $=.33$ y $\omega=.79)$} \\
\hline 5. Cuando me venía a la cabeza el problema, trataba de concentrarme en otras cosas & .72 & .66 \\
\hline 26. Procuré no pensar en el problema & .68 & .65 \\
\hline 12. Me volqué en el trabajo o en otra actividad para olvidarme del problema & .64 & .67 \\
\hline 19. Salí al cine, a cenar, a «dar una vuelta», etc., para olvidarme del problema & .37 & .45 \\
\hline 33. Practiqué algún deporte para olvidarme del problema & .33 & .41 \\
\hline
\end{tabular}

Notas. Método de extracción: factorización de eje principal. Método de rotación: Promax con normalización Kaiser. Cargas: cc = carga configuracional o saturación en la matriz de configuraciones y ce = carga estructural o saturación en la matriz estructural. $\alpha=$ coeficiente de consistencia interna alfa de Cronbach, AVE = varianza media extraída y $\omega$ = fiabilidad compuesta u omega de McDonald. El AVE y $\omega$ se calcularon a partir de las cargas estructurales.

* (C) Sandín y Chorot (2003, p. 52). Reproducido con permiso de los autores. 
18, 25 y 32) para medir EEA alcanzó buen ajuste por los nueve índices una vez que se definió la correlación entre los residuos de los ítems 25 y 32 , todas sus estimaciones fueron significativas por PCS y cumplió con los dos criterios de validez convergente $(\mathrm{AVE}=.53 \mathrm{y} \omega=.85)$. El modelo para medir EVT tuvo buen ajuste por siete índices y aceptable por dos una vez liberada la correlación entre los residuos de los ítems 5 y 33, todas sus estimaciones fueron significativas por PCS (ítems 5, 12, 19, 26 y 33) y cumplió con el criterio de la fiabilidad compuesta para la validez convergente $(\omega=.72)$, pero no con el de la varianza media extraída $(\mathrm{AVE}=.35)$. El modelo para medir FSP tuvo buen ajuste por ocho índices y aceptable por uno una vez que se especificaron dos correlaciones entre residuos (de los ítems 8 y 36 y de los ítems 1 y 15), todas sus estimaciones fueron significativas por PCS (ítems 1, 8, 15, 29 y 36) y cumplió con los dos criterios de validez convergente (AVE $=.62$ y $\omega=$ .89). El modelo para medir RLG tuvo un ajuste bueno por tres índices ( $p$ de B-S, CFI y SRMR), adecuado por cuatro $\left(\chi^{2}, \chi^{2} / g l\right.$ GFI y NNFI), aunque malo por dos (AGFI y RMSEA) una vez que se liberaron tres correlaciones entre tres residuos de los ítems 28, 35 y 42 (factor específico subyacente de ayuda divina a través del rezo), todas sus estimaciones fueron significativas por PCS (ítems $7,14,21,28,35$ y 42) y cumplió con los dos criterios de validez convergente $(\mathrm{AVE}=.60 \mathrm{y} \omega=.90)$. Al hacer el contraste por mínimos cuadrados no ponderados $($ MSMK $=35.04>10)$, el ajuste del modelo con tres correlaciones entre residuos fue bueno ( $p$ de $\mathrm{B}-\mathrm{S}=.168$, $\chi^{2} / g l=1.941 / 6=0.324, \mathrm{GFI}=.998, \mathrm{AGFI}=.994, \mathrm{NFI}=$ .997 , RFI $=.993$ y SRMR $=.034$ ), y todas sus estimaciones significativas por PCS. El modelo de un factor para medir BAS tuvo buen ajuste por los nueve índices una vez que se introdujo la correlación entre los residuos de los ítems 27 y 41 , todas sus estimaciones fueron significativas por PCS (ítems 6, 13, 20, 27, 34 y 41), y cumplió con los dos criterios de validez convergente $(\mathrm{AVE}=.61$ y $\omega=.90)$ (Tabla 1$)$.

Para definir el modelo de la relación entre las puntuaciones de los seis factores (Modelo M2O), se partió del resultado del AFE de segundo orden que arrojó tres factores: afrontamiento emocional negativo (AEN) con tres indicadores (-FSP, +AFN y +EEA), apoyo social (ASO) con dos indicadores (BAS y EEA) y afrontamiento evitativo/religiosidad (AER) con dos indicadores (EVT y RLG), presentando correlación negativa AEN y AER. En primer lugar, se especificó el factor de AEN con sus tres indicadores. Debido a un número insuficiente de indicadores, el factor de AER quedó especificado como la correlación entre dos variables exógenas (EVT y RLG). Para representar en el modelo el factor de ASO, se intro- dujo BAS como una variable exógena, y se especificó la relación direccional de BAS hacia la variable endógena EEA. Inicialmente se contempló la correlación del factor de AEN con las variables EVT y RLG. Al no ser significativas estas correlaciones por PCS $\left(r_{[\mathrm{AEN}, \mathrm{EVT}]}=.051\right.$, IC del 95\%: $[-.29, .35], p=.764 ; r_{[\mathrm{AEN}, \mathrm{RLG}]}=-19$, IC del 95\%: $[-.01, .39], p=.062)$ y tras revisar los índices de mejora de ajuste, se especificó la relación direccional de la variable exógena EVT hacia la variable endógena FSP (Figura 1). El modelo presentó todas sus estimaciones significativas por PCS. El ajuste fue bueno por cuatro índices y aceptable por cuatro (Tabla 1 y Figura 1).

Figura 1. Modelo de ecuaciones estructurales para representar la relación entre las escalas revisadas del CAE (M2O).

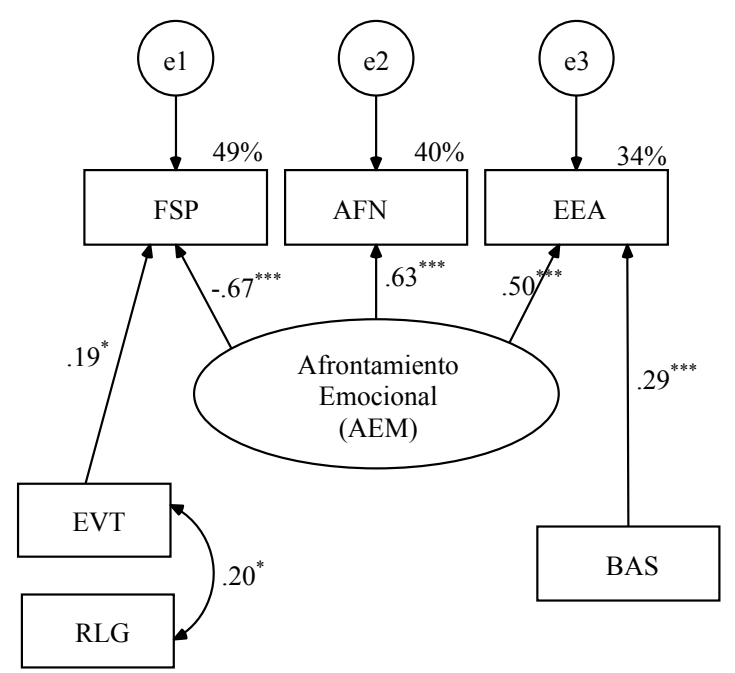

Nota. Significación: contraste bilateral: $* p \leq .05, * * * p \leq .001$.

Perfil promedio en estrategias y estilos de afrontamiento

Con la configuración final de seis factores y con base en la media aritmética, se encontró que las estrategias de afrontamiento entre los participantes de la muestra siguieron el siguiente orden: FSP con una media de 2.42 $(D T=0.95)$ en el intervalo $[2.4,3.2)$ correspondiente a «con frecuencia»; EVT con una media de 1.94 ( DT = $0.74)$ en el intervalo $[1.6,2.4)$ correspondiente «a veces»; $\operatorname{BAS}(M=1.59 ; D T=0.99), \operatorname{AFN}(M=1.39 ; D T=$ $0.74)$ y $\operatorname{EEA}(M=1.20 ; D T=0.83)$ en el intervalo $[0.8$, 1.6) correspondiente a «muy pocas veces»; y RLG $(M=$ $0.58 ; D T=0.87)$ en el intervalo $[0,0.8)$ correspondiente a «nunca».

Se calculó las puntuaciones en los estilos en un rango continuo y homogéneo de 0 a 4 para poderlas comparar: $\mathrm{AEN}=(20-\mathrm{FSP}+\mathrm{AFN}+\mathrm{EEA}) / 17, \mathrm{ASO}=(\mathrm{BAS}+$ 
EEA $) / 11, y$ AER $=(E V T+R L G) / 11$. Por la prueba ómnibus de D'Agostino-Pearson las distribuciones de AEN $(D P=2.26, p=.322)$ y ASO $(D P=2.97, p=.227) \mathrm{se}$ ajustaron a una distribución normal. La distribución de AER mostró asimetría positiva ( $\mathrm{Sk}=1.24$, IC del 95\%: $0.58,1.70)$ y leptocurtosis $(K=2.03$, IC del $95 \%: 0.02$, 4.10). La comparación global de media por un análisis de varianza de medidas repetidas mostró diferencia significativa (prueba de efectos intraparticipantes con la corrección de Greenhouse-Geisser: $F_{[1.86,184.23]}=3.65, \mathrm{p}$ $=.031$, al no asumirse independencia y homocedasticidad de residuos por la prueba de esfericidad de Mauchly: $\chi_{[2]}^{2}=7.61, p=.022$, siguiendo los residuos de AEN y ASO una distribución normal, aunque los de AER mostraron asimetría positiva y leptocurtosis). En las comparaciones a posteriori por pares, la media en AER $(M=1.20, D T=0.63)$, en comparación con las medias en ASO $(M=1.42, D T=0.73)$ y AEN $(M=1.39$, $D T=0.62)$, fue significativamente menor $(t[99]=2.28$, $p=.025$ y $t[99]=2.10, p=.039$, respectivamente).

\section{Discusión}

En relación con el primer objetivo de contrastar el modelo original, se llevó a cabo un AFC, donde los malos índices de ajuste, los parámetros no significativos, y la consistencia interna inaceptable en REP, alertaron de que la solución factorial no fuese adecuada para la población trans y se exploró una nueva. Precisamente, se había propuesto como tercer objetivo explorar un modelo alterno.

En relación con el segundo objetivo de cálculo de la consistencia interna, el cuestionario de 42 ítems presentó una buena consistencia interna, pero no así todos sus factores. La escala REP presentó un coeficiente inaceptable y EVT cuestionable. En los estudios realizados en México (González y Landero, 2007; Moral y Martínez, 2009; Moral y Miaja, 2012), la escala de EVT presentó coeficientes cuestionables, y la escala de REP tuvo problemas de fiabilidad. Otra escala que reveló problemas en estos estudios fue AFN, cuyo indicador fue aceptable en la presente investigación.

Se eliminaron ocho ítems con base en el AFE de los siete factores originales y el análisis de consistencia interna de los factores. En relación con el tercer objetivo de explorar la estructura factorial del CAE, los resultados del AFE de primer orden con los 34 ítems, mostraron que la estructura propuesta por Sandín y Chorot (2003) no se reprodujo exactamente. Se encontraron seis factores, número que se sustentó por el criterio de Velicer, que permite retener factores con varianza sustantiva (Courtney, 2013). Los seis factores explicaron el 63.8\% de la varianza total, cifra superior a la reportada por Sandín y Chorot $(55.3 \%)$ con siete factores. Cuatro factores mostraron validez convergente (AVE $>.50$ y $\omega>.70$ ), pero el resto (EVT y AFN) sólo cumplieron con el segundo criterio de validez convergente $(\omega>.70)$, por lo que su validez convergente fue algo débil. No obstante, sí presentaron validez discriminante conforme a la expectativa (González y Landero, 2007; Moral y Martínez, 2009; Moral y Miaja, 2012; Sandín y Chorot, 2003).

Un resultado inesperado fue la significativa reducción de los indicadores de la escala de REP, y la inclusión con carga negativa de sus dos ítems restantes junto a los ítems de la escala de AFN. Un aspecto a destacar es la ausencia de la reevaluación positiva en la presente muestra. Una posible explicación a lo anterior puede encontrarse a través del concepto de estrés minoritario de Meyer (2003). Este concepto se utiliza específicamente para distinguir el estrés excesivo al cual están expuestas las personas de una categoría social estigmatizada como resultado de su posición social como minoría. Estos estresores minoritarios incluyen experiencias de discriminación y/o violencia, los cuales a su vez conllevan a expectativas de rechazo, vigilancia, ocultamiento de la identidad de género o internalización de actitudes sociales negativas (homofobia internalizada). De esta forma, si una persona experimenta discriminación por su identidad de género u orientación sexual, es probable que incremente su vigilancia, y tenga expectativas de rechazo en futuras situaciones.

En este sentido, Allport (1979) describió que las personas objeto de prejuicio desarrollan la vigilancia como una estrategia de afrontamiento defensiva. Las personas no heterosexuales y trans aprenden a anticipar tratos negativos de parte de miembros de la cultura dominante (en este caso personas heterosexuales y cisgénero [persona cuyo sexo asignado al nacer corresponde con su identidad de género]), por lo que para evitar potenciales tratos negativos, como discriminación y violencia, deben estar vigilantes. Consecuentemente, tal vigilancia es crónica, ya que se repite de manera continua en la vida cotidiana de la persona minoritaria.

Cabe mencionar que las personas de la diversidad sexual han reportado una mayor prevalencia de trastornos mentales debido al estrés excesivo (Dohrenwend, 2000). Se ha encontrado una mayor prevalencia de síntomas depresivos e intentos de suicidio en población trans que en población cisgénero ( $\mathrm{Su}$ et al., 2016). Lo anterior podría explicar la aparición del factor AFN con ítems con carga negativa de REP en esta muestra.

Como siguiente análisis se realizó un AFE de segundo orden, donde los resultados no reprodujeron la estructura de dos factores propuesta para el CAE (Sandín y Chorot, 2003). Este análisis reveló tres factores de or- 
den superior, número sustentado por la convergencia de los cinco criterios contemplados, lo que da gran fuerza al resultado (Courtney, 2013). Los tres factores explicaron un $70.2 \%$ de la varianza total, la cual fue superior al $43.6 \%$ reportado por González y Landero (2007), al $56.5 \%$ por Moral y Miaja (2012) y al $49.3 \%$ en el estudio de Sandín y Chorot (2003).

El primer factor de segundo orden denominado afrontamiento emocional negativo (-FSP, +AFN y +EEA) hace alusión a un factor de afrontamiento desadaptativo, ya que las formas de afrontamiento que se describen en sus ítems no llevan a la persona a encontrar una solución al problema, sino a expresar ira y pensar de forma pesimista y derrotista. En el segundo factor de segundo orden se encontró la escala de BAS, lo que pudiera considerarse como un factor de locus de control externo, donde la persona acude a sus redes cercanas con la intención de ser reconfortada, comprendida y aconsejada. El tercer factor de evitación y religiosidad muestra que la persona no realiza ninguna acción para solucionar el problema y espera que éste se resuelva por intervención de un ser superior. Por lo antes mencionado, no se logró identificar ningún estilo de afrontamiento que pueda ser considerado claramente adaptativo.

Cuando se forzó la solución a dos factores para probar el modelo de Sandín y Chorot (2003), se obtuvo un resultado análogo en su interpretación al de los autores españoles, aunque no en su configuración. En el primer factor de segundo orden se encontraron las estrategias de AFN y EEA (afrontamiento emocional), y en el segundo FSP y BAS (afrontamiento activo). Las escalas de EVT y RLG quedaron fuera de ambos factores, ya que requieren la emergencia de uno tercero para su correcta ubicación. El primer factor encontrado implica emociones negativas (ira, ansiedad, tristeza), así como consecuencias negativas debidas a la irritabilidad y desmotivación. El factor de afrontamiento activo es análogo al de afrontamiento adaptativo de Sandín y Chorot (2003), ya que implica buscar soluciones y buscar asesoramiento y apoyo para alcanzar éstas. Esta interpretación de afrontamiento desaptativo versus adaptativo viene reforzada por la correlación moderada y negativa entre ambos factores (González y Landero, 2007).

A un nivel exploratorio, se comprobó el ajuste a los datos del modelo con tres factores de orden superior, usando AFC. Se usa el adjetivo exploratorio y el verbo comprobar, y no se habla de confirmar, ya que esto requeriría una muestra independiente. Finalmente, se representó por medio de un modelo estructural con buen ajuste a los datos en términos generales. Se especificó un factor latente de afrontamiento emocional negativo con tres indicadores (baja focalización en la solución de pro- blemas, autofocalización negativa y expresión abierta de irritabilidad y nerviosismo). El factor de apoyo social se representó como una relación predictiva directa de la búsqueda de apoyo social hacia la expresión emocional abierta, indicando extroversión y exteriorización del conflicto o el estrés. El factor de evitación y religiosidad se representó por la correlación entre estas dos escalas, apareciendo una relación predictiva directa de la evitación hacia la focalización en la solución del problema, indicando que se trata de aspectos de introversión, interiorización y reflexión, es decir, el polo opuesto al anterior. Finalmente, este modelo habla de emoción negativa, exteriorización e interiorización, lo que asemeja a los modelos de segundo orden con las escalas clínicas del MMPI-2 (Moral, 2014).

Se enunció como cuarto objetivo del estudio describir el perfil promedio de afrontamiento. Los datos revelaron que dentro de las estrategias de afrontamiento más utilizadas en esta muestra de personas trans se encuentra la FSP, seguida de EVT, BAS y AFN. Dentro de las estrategias menos utilizadas se encontraron la EEA y RLG.

Dependiendo de la controlabilidad del estresor el afrontamiento enfocado al problema es predominante cuando la persona siente que la situación es controlable o modificable, mientras que el enfocado a la emoción es predominante cuando la persona siente que no tiene control sobre el evento estresante (Folkman y Lazarus, 1980; Scheier, Weintraub y Carver, 1986). Con base en lo anterior, el presente estudio sugiere que aquellos eventos interpretados como susceptibles de cambio (empleando la solución de problemas) pudieran corresponder a aquellos eventos referentes al proceso de transición en las personas trans y que aquellos no susceptibles de cambio (empleo de autofocalización negativa, evitación, expresión emocional y religión) podrían corresponder a experiencias de estigma y/o discriminación.

En la revisión de la literatura no se encontró información sobre el factor EEA (irritabilidad) en población trans; no obstante, en personas LGB se ha encontrado que el ocultamiento de la orientación sexual, además de suprimir emociones, previene a las personas LGB de desarrollar su identidad y afiliarse con otras personas de la comunidad, lo que repercute en la disminución de su salud y pérdida de los efectos protectores por haber «salido del closet» (Meyer, 2003). Carver et al. (1989) proponen que la búsqueda de apoyo social puede ser un puente entre tendencias funcionales y no funcionales. Sus resultados mostraron que la búsqueda de apoyo social se asoció con un afrontamiento activo y planeación, pero también con expresión emocional, lo cual a su vez está relacionado con negación y distanciamiento. En el presente estudio la búsqueda de apoyo social se asoció a 
expresión emocional abierta (irritabilidad) en un sentido de exteriorización del estrés y el conflicto.

La religiosidad fue la estrategia de afrontamiento menos frecuente. Esto se puede atribuir a que la religión se ha utilizado para legitimar la exclusión de personas LGBT en la sociedad, al acudirse a algunos textos doctrinales que sustentan que su atracción y sexualidad son pecaminosas y auguran un castigo divino; asimismo, la religión se ha utilizado para monitorear y controlar el comportamiento e identidad de personas de la diversidad sexual (Halkitis et al., 2009). Por lo anterior no es inusual que poblaciones de minorías sexuales abandonen la religión de su infancia (Rosario, Yali, Hunter y Gwa$\mathrm{dz}, 2006)$ o sean menos religiosos que la población general (Barnes y Meyer, 2012).

El estudio posee varias limitaciones. En primer lugar, el número de participantes es demasiado bajo, tanto para la realización del AFE como del AFC y MEE. No obstante, debido a la falta de estudios en este tipo de población y la gran dificultad para obtener muestras más grandes, se consideró pertinente realizar tales tipos de análisis, los cuales deben ser considerados meramente exploratorios y provisionales. En relación a la identidad de género de los participantes, otra de las debilidades consistió en la falta de equivalencia de los grupos de hombres trans y mujeres trans, por lo que no se descarta que los resultados puedan estar mayormente influenciados por los hombres trans, quienes representan el $79 \%$ de la muestra. De igual manera, la anterior limitante no permitió realizar una comparación entre los dos grupos. Otra limitación fue el uso de un muestreo intencional. No obstante, la técnica de bola de nieve y el responder la encuesta sin ser observado (por internet), parecer constituir la mejor opción ante poblaciones ocultas y estigmatizadas (Ritter, Loring, Laurent, y Mathews, 2004).

En conclusión, la versión reducida a 34 ítems presenta una consistencia interna global aceptable y una estructura de seis factores. Dos factores presentan una consistencia interna excelente, tres buena, pero la del factor de evitación fue cuestionable. En el AFE, todos cuentan con validez convergente, salvo evitación, y validez discriminante. A estos seis factores de primer orden subyacen tres factores de orden superior: afrontamiento emocional negativo, apoyo social y afrontamiento evitativo/religiosidad. Un modelo de un factor de afrontamiento emocional negativo con tres indicadores (FSP con carga negativa y AFN y EEA con cargas positivas), las escalas de religión y evitación correlacionadas (afrontamiento evitativo/religiosidad) con la evitación como determinante de focalización en la solución de problemas (introversión/interiorización/reflexión) y búsqueda de apoyo social como determinante de expresión emocional abierta (extroversión/exteriorización), constituyen una representación del modelo factorial de segundo orden con buen ajuste a los datos. En el perfil promedio de afrontamiento, FSP se emplea con frecuencia, EVT a veces, BAS, AFN y EEA muy pocas veces y RLG nunca, además domina el estilo BAS/EEA o de exteriorización sobre el estilo EVT/RLG o de interiorización.

Debido al tamaño muestral limitado, se recomienda usar las presentes conclusiones como hipótesis a confirmar con una muestra más grande, de al menos 400 participantes (200 mujeres trans y 200 hombres trans). También se sugiere estudiar su fiabilidad temporal y la estabilidad de su estructura factorial mediante un diseño de cohorte.

\section{Conflictos de intereses}

Los autores declaran no tener ningún conflicto de intereses. El tercer autor es miembro de la organización no gubernamental Universitarios por la Equidad y una Sociedad Integra e Incluyente (UNESII).

\section{Referencias}

Agius, S., \& Tobler, C. (2012). Trans and Intersex People. Discrimination on Grounds of Sex, Gender Identity and Gender Expression. Luxemburgo: Unión Europea.

Allport, G. (1979). The nature of prejudice. Reading, MA: Addison-Wesley.

American Psychological Association (2017). Ethical principles of psychologists and code of conduct. With the 2016 amendment to standard 3.04. Washington, DC: Author.

Amirkhan, J. H. (1990). A factor analytically derived measure of coping: the coping strategy indicator. Journal of Personality and Social Psychology, 59, 1066-1074. doi: 10.1037//00223514.59.5.1066

Barnes, D., \& Meyer, I. (2012). Religious affiliation, internalized homophobia, and mental health in lesbians, gay men, and bisexuals. American Journal of Orthopsychiatry, 82, 505-515. doi: 10.1111/j.1939-0025.2012.01185.x

Barrios, D. (2014). Resignificar lo masculino. Guía de supervivencia para varones del siglo XXI. Ciudad de México: Vila Editores.

Budge, S. L., Katz-Wise, S. L., Tebbe, E. N., Howard, K. A. S., Schneider, C. L., \& Rodriguez, A. (2013). Transgender emotional and coping processes. The Counseling Psychologist, 41, 601-647. doi:10.1177/0011000011432753

Byrne, B. (2016) Structural equation modeling with AMOS. Concepts, applications, and programming (3rd ed.). New York: Routledge.

Carrillo, M. F. (2008). Transgresión desde adentro: El caso del reconocimiento jurídico de las personas transgénero y transexuales en Ciudad de México. Tesis de maestría sin publicar. Facultad Latinoamericana de Ciencias Sociales FLACSO sede México. Recuperado de http:// conocimientoabierto.flacso.edu.mx/tesis/229 
Carver, C. S., Scheier, M. F., \& Weintraub, J. K. (1989). Assessing coping strategies: A theoretically based approach. Journal of Personality and Social Psychology, 56, 267-283. doi: 10.1037/0022-3514.56.2.267

Courtney, M. G. R. (2013). Determining the number of factors to retain in EFA: Using the SPSS R-Menu v2.0 to make more judicious estimations. Practical Assessment Research and Evaluation, 18, 24-57. Recuperado de http://pareonline.net/ getvn.asp? $=18 \& \mathrm{n}=8$

Cronbach, L. J., \& Shavelson, R. J. (2004). My current thoughts on coefficient alpha and successor procedures. Educational and Psychological Measurement, 64, 391-418. doi: 10.1037/ e645652011-001

Dohrenwend, B. (2000). The role of adversity and stress in psychopathology: Some evidence and its implications for theory and research. Journal of Health and Social Behavior, 41, 1-19. doi: 10.2307/2676357

Endler, N. S., \& Parker, J. D. A. (1990). Multidimensional assessment of coping: a critical evaluation. Journal of Personality and Social Psychology, 58, 844-854. doi: 10.1037//0022-3514.58.5.844

Folkman, S., \& Lazarus, R. (1980). An analysis of coping in a middle-aged community sample. Journal of Health and Social Behavior, 21, 219-239. doi: 10.2307/2136617

González, M. T., y Landero, R. (2007). Cuestionario de Afrontamiento del Estrés (CAE): validación en una muestra mexicana. Revista de Psicopatología y Psicología Clínica, 12(2), 189-198. doi: 10.5944/rppc.vol.12.num.3.2007.4044

Halkitis, P., Mattis, J., Sahadath, J., Massie, D., LAdyzhenskaya, L., Pitrelli, K. ... Cowie, S. (2009). The meanings and manifestations of religion and spirituality among lesbian, gay, bisexual and transgender adults. Journal of Adult Development, 16, 250-262. doi: 10.1007/s10804-009-9071-1

Hooper, D., Coughlan, J., \& Mullen, M. R. (2008). Structural equation modelling: Guidelines for determining model fit. Electronic Journal of Business Research Methods, 6, 53-60.

Lazarus, R. S., \& Folkman, S. (1991). Estrés y procesos cognitivos. Barcelona: Martínez Roca.

Lloret, S., Ferreres, A., Hernández, A., y Tomás, I. (2014). El análisis factorial exploratorio de los ítems: una guía práctica, revisada y actualizada. Anales de Psicología, 30, 1151-1169. doi: 10.6018/analesps.30.3.199361

Malhotra, N. K. \& Dash, S. (2011). Marketing research: an applied orientation (6 $6^{\mathrm{a}}$ ed.). New Delhi: Pearson Education.

Meyer, I. (2003). Prejudice, social stress, and mental health in lesbian, gay, and bisexual populations: Conceptual issues and research evidence. Psychological Bulletin, 129, 674-697. doi: 10.1037/0033-2909.129.5.674
Molina, N. E., Guzmán, O. O., y Martínez, A. (2015). Identidades transgénero y transfobia en el contexto mexicano. Quaderns de psicologia, 17, 71-82.

Moos, R. H. (1993). Coping responses inventory. Florida, USA: Psychological Assessment Resources.

Moral, J. (2014). Dimensiones psicopatológicas de las escalas clínicas del MMPI-2. Revista de Psicopatología y Psicología Clínica, 19(1), 45-62. doi:10.5944/rppc.vol.19.num.1.2014.12982

Moral, J. y Martínez, J. (2009). Reacción ante el diagnóstico de cáncer en un hijo: estrés y afrontamiento. Psicología y Salud, 19, 189-196.

Moral, J., y Miaja (2012). Validación del Cuestionario de Afrontamiento del Estrés en dos muestras de seropositivos y población general. Ansiedad y Estrés, 18, 15-29.

Peñacoba, C. y Moreno, B. (1999). La escala de estresores universitarios (EEU) Una propuesta para la evaluación del estrés en grupos poblacionales específicos. Ansiedad y Estrés, 5, 61-78.

Ritter, P., Lorig, K., Laurent, D., \& Matthews, K. (2004). Internet versus mailed questionnaires: A randomized comparison. Journal of Medical Internet Research, 6, e29. doi: 10.2196/jmir.6.3.e29

Rosario, M., Yali, A., Hunter, J., \& Gwadz, M. (2006). Religion and health among lesbian, gay, and bisexual youths: An empirical investigation and theoretical explanation. En A. Omato \& H. Kurtzman(Eds), Sexual orientation and mental health: Examining identity and development in lesbian, gay and bisexual people (pp. 117-140). Washington, DC: American Psychological Association.

Peña, E. Y. (2014). Transexualidad: ¿patología, identidad o proceso? Estudios de Antropología Sexual, 1, 68-88. Recuperado de https://revistas.inah.gob.mx/index.php/antropologiasexual/ article/view/574

Sandín, B. (2003). El estrés: un análisis basado en el papel de los factores sociales. International Journal of Clinical Health Psychology, 3, $141-157$.

Sandín, B. y Chorot, P. (2003). Cuestionario de afrontamiento del estrés (CAE): desarrollo y validación preliminar. Revista de Psicopatología y Psicología Clínica, 8, 39-54. doi: 10.5944/ rppc.vol.8.num.1.2003.3941

Scheier, M. E, Weintraub, J. K., \& Carver, C. S. (1986). Coping with stress: Divergent strategies of optimists and pessimists. Journal of Personality and Social Psychology, 51, 1257-1264. doi: 10.1037//0022-3514.51.6.1257

Stryker, S. (2015). Transgender. GLBTQ Archive. Recuperado de http://www.glbtqarchive.com/ssh/transgender_S.pdf

Su, D., Irwin, J., Fisher, C., Ramos, A., Kelley, M., Rogel, D., \& Coleman, J. (2016). Mental health disparities within the LGBT population: A comparison between transgender and nontransgender individuals. Transgender Health, 1, 12-20. doi: $10.1089 / \operatorname{trgh} .2015 .0001$ 